sehr befriedigendes Unterfangen. Wer lieber in einer Kanzlei arbeiten möchte, sollte sich eine suchen, die wie Weil Gotshal und Manges global auftritt und extrem hohe Ansprüche hat und sich um den Nachwuchs kümmert. Matt Powers unterstützt mich noch heute - ich werde ihm dafür immer dankbar sein.

\title{
Leiterin der Geschäftsstelle des Deutschen Notarvereins in Brüssel
}

\author{
Prof. Dr. Stephanie Michel \\ Schatzmeisterin der Regionalgruppe Brüssel im djb; Rechtsan- \\ wältin, Brüssel
}

Bevor ich mich für ein juristisches Studium entschlossen hatte, galt meine Leidenschaft noch der Musik. Nach dem Abitur begann für mich ein sechsmonatiges Volontariat beim Schleswig-Holstein-Musik-Festival in Hamburg, dem ein Studienprogramm für das Fach Klavier am Konservatorium in Paris folgte. Erst mit 22 Jahren habe ich zur Freude meiner Eltern beschlossen, mich dieser Neigung nur noch in meiner Freizeit zu widmen und an der Universität Göttingen mein Studium der Rechtswissenschaften begonnen. Nach einem Wechsel an die Universität München und einem weiteren Wechsel an die Universität Hannover habe ich nach sechs Semestern die erste juristische Staatsprüfung abgelegt. Mir war durchaus bewusst, dass die Studienzeit von sechs Semestern kurz bemessen war. Aber nachdem die schriftlichen Ergebnisse recht passabel waren, ging ich guter Dinge zur mündlichen Prüfung. Nicht bewusst war mir, dass die erst kurz zuvor eingeführte Freischussregelung bei zahlreichen Professoren auf heftige Kritik gestoßen war und sämtliche Prüfer in meiner Examensprüfung keinen Zweifel daran ließen, dass sie zu diesen gehörten. Dieser Tag war sehr einschneidend, hatte aber zum Glück keinen weiteren Einfluss auf meinen beruflichen Weg.

Im Anschluss an das erste Staatsexamen ergab sich die Möglichkeit, als Repetitorin für Wegner \& Abels zu beginnen; in den drei folgenden Jahren habe ich in Hannover und Göttingen Zivilrecht unterrichtet. Der damaligen Geschäftsführerin bin ich noch heute dankbar für ihr Vertrauen in meine Fähigkeiten, denn dies war durch die Erfahrung der mündlichen Prüfung im ersten Staatsexamen sehr erschüttert. Durch die Dozententätigkeit habe ich viel gelernt und meine Leidenschaft für das Unterrichten entdeckt.

Das Referendariat habe ich im Landgerichtsbezirk Frankfurt am Main absolviert. Während der Anwaltsstation konnte ich wertvolle Erfahrungen in einer amerikanischen Großkanzlei in New York sammeln. Mein Berufswunsch stand fest: Rechtsanwältin. Während der Referendariatszeit absolvierte ich den theoretischen Teil der Kurse zum Fachanwalt für Arbeitsrecht. Ich hatte das große Glück, dass die Vortragenden aus diesem Kurs zu den besten Arbeitsrechtlern zählten und darüber hinaus noch einen unvergesslichen Vortragsstil beherrschten. Das größte Glück war aber, dass ich den Dozenten
Prof. Dr. Peter Schwerdtner kennenlernen durfte, der dann später auch mein Doktorvater wurde. Nach Abschluss meines zweiten Staatsexamens im Dezember 1998 habe ich unverzüglich mit der Doktorarbeit begonnen. Da das Thema vier brandaktuelle Entscheidungen des Bundesarbeitsgerichts aus dem Jahr 1997 zur Grundlage hatte, gab es kaum Literatur zu dem Thema, so dass zeitaufwendige Recherchearbeiten nicht erforderlich waren und die Arbeit in sechs Monaten fertiggestellt werden konnte. Die größte Schwierigkeit an der Doktorarbeit bestand darin, einen Gesprächstermin bei meinem sehr geschätzten Doktorvater zu erhalten. Ich erinnere mich gern an Reisen nach Frankfurt und Hamburg, nur um mit ihm auf der Zugfahrt Gelegenheit zu haben, über die Arbeit zu sprechen. Aber für diese Gelegenheit eines Gespräches mit einem derart überragenden Juristen und Menschen wäre ich auch noch weiter gereist. Auch wenn wir nie wirklich über meine Arbeit gesprochen haben, sondern ich eher darin getestet wurde, ob ich auch nach fünf Glas Wein der jeweiligen Region noch die zuvor überlegten Fragen einwandfrei formulieren konnte. Ich konnte es nicht und habe bei der letzten Fahrt auch gleich sämtliche Unterlagen im Zug liegen lassen. Es war herrlich.

Nach Abschluss der Doktorarbeit habe ich in der Hannoveraner Kanzlei Stobbe: Rechtsanwälte begonnen. Die Kanzlei hat in Hannover eine lange Tradition und zählte zu den größten Kanzleien der Stadt. Nachdem die Kanzlei ganze 72 Jahre - nämlich seit Gründung im Jahre 1927 - ohne auch nur eine einzige Frau als Rechtsanwältin bestanden hatte, gab es mit meiner Einstellung die erste weibliche Kollegin in dieser Herrenrunde. An dieser Stelle sei erwähnt, dass nach meinem Weggang weitere Frauen in der Kanzlei als Rechtsanwältinnen eingestellt wurden. Meine Tätigkeit als Repetitorin habe ich während dieser Zeit aufgegeben und dafür im Jahr 2001 einen Lehrauftrag für Wirtschaftsrecht an der FHDW in Hannover angenommen. Meine Kanzlei hat diese Nebentätigkeit sehr unterstützt, so dass ich zwei Nachmittage in der Woche jeweils vier Stunden unterrichten konnte. Die FHDW ist eine private Fachhochschule in Hannover, unter anderem für die Fächer Betriebswirtschaft und Wirtschaftsinformatik. Ich treffe immer wieder ehemalige Student(inn)en in den verschiedensten Bereichen und freue mich zu sehen, dass sie überwiegend beeindruckende Karrieren machen. Ich traf einmal einen ehemaligen Studenten, der mir sagte, dass er bei mir trotz seiner Abneigung gegen die Jurisprudenz sehr viel gelernt hätte. 
Zu der Zeit verschickte Microsoft gerade Schreiben an viele Unternehmen in Deutschland, die keine Lizenzen von Microsoft erworben hatten, das mit dem Satz begann: „Wie erklären Sie sich, dass ein Unternehmen Ihrer Größe keine Lizenzen von Microsoft registriert hat? “ Der Student kam aus einem großen Bäckereibetrieb mit zahlreichen Filialen in mehreren Städten, nennen wir sie mal Meiser. Stolz sagte er, er habe zurückgeschrieben: „Und wie erklären Sie sich, dass ein Unternehmen in der Größe von Microsoft seine Brötchen nicht bei Bäckerei Meiser kauft? “ Zumindest hat Microsoft diese Angelegenheit nicht weiterverfolgt.

Im Jahr 2002 haben die Sozien meiner Rechtsanwaltskanzlei mir die Aufnahme in die Sozietät angeboten. Das war für mich eine große Ehre, nicht nur, weil es sich um eine hervorragende Rechtsanwaltskanzlei handelte, vielmehr weil ich jeden Einzelnen der Kollegen so sehr schätze. Aus beruflicher Sicht konnte ich es mir nicht besser vorstellen, aber der Blick auf mein Privatleben ließ mich sehr daran zweifeln, eine derartig feste Bindung mit einer Sozietät einzugehen. Denn eigentlich habe ich in dieser bewegten Zeit als Rechtsanwältin mit der Nebentätigkeit als Lehrbeauftragte eines ganz aus den Augen verloren: Mein größter Wunsch war es, eine Familie zu gründen. Schweren Herzens habe ich die Partnerschaft abgelehnt und die Rechtsanwaltstätigkeit vorübergehend aufgegeben. Ich wollte zeitlich etwas flexibler sein und das Arbeitsrecht auch aus Unternehmenssicht kennenlernen. Nach einigen Monaten, in denen ich Führungskräfteschulungen im Arbeitsrecht in zahlreichen Unternehmen in Deutschland durchgeführt habe, nahm ich eine Tätigkeit in der Hannover Rückversicherung AG in Hannover an. In den zwei Jahren, die ich dort verbracht habe, konnte ich bei einem international tätigen Unternehmen sehr gut sehen, wie Unternehmensentscheidungen getroffen werden und arbeitsrechtliche Vorgänge in der Praxis funktionieren.

Im Jahr 2004 wurde dann aus meinem Lehrauftrag an der FHDW eine Professur. Zahlreiche Veröffentlichungen ebneten diesen Weg. Und auch an der FHDW ist ein Lehrstuhl zum ersten Mal mit einer Professorin besetzt worden.

Im Jahr 2004 ging mein Mann beruflich nach Brüssel. Die Entscheidung, ihm zu folgen, stand fest. Wir bekamen das erste Kind und es folgten in den nächsten drei Jahren noch zwei weitere Kinder. Meine berufliche Tätigkeit beschränkte sich in dieser Zeit auf Veröffentlichungen und die Bearbeitung von zahlreichen Diplomarbeiten. Einige Male im Jahr reiste ich nach Hannover, um mündliche Prüfungen an der FHDW abzunehmen. Die Zeit mit den Kindern war wunderbar, aber ich merkte, dass mir die Berufswelt fehlte. Mit drei kleinen Kindern kam für mich nur eine Teilzeittätigkeit in Frage. Eine solche zu finden, die dann auch noch den Vorstellungen entspricht, war nicht einfach. Während meiner Zeit in Brüssel wurde ich Mitglied des djb der Regionalgruppe Brüssel. Die zahlreichen und sehr interessanten Veranstaltungen waren immer eine gelungene Abwechslung zu dem Leben zu Hause, welches mit den kleinen Kindern keine einzige Gelegenheit bot, auch nur einen klaren Gedanken zu fassen. Als ich in der Regionalgruppe den Wunsch nach einer beruflichen Tätigkeit äußerte, habe ich von einigen interessanten Stellen erfahren. Ich habe meine heutige Stelle der ehemaligen stellvertretenden Vorsitzenden des djb zu verdanken, die mir eine noch nicht öffentliche Stellenausschreibung weiterleitete. Der Deutsche Notarverein suchte eine Leiterin des Brüsseler Büros in Teilzeit. Auf diese Stelle habe ich mich beworben und zwei Wochen vor der Entbindung meines dritten Kindes das Bewerbungsgespräch geführt. Nur wenige Monate später habe ich im Jahr 2008 die Stelle angetreten. Meine Entscheidung, im Jahr 2009 dem Vorstand der Regionalgruppe des djb beizutreten, bekundet unter anderem den Dank, den ich durch mehr eigenes Engagement in dieser Gruppe zum Ausdruck bringen möchte. Die Vertretung der Interessen des Deutschen Notarvereins vor den Institutionen der Europäischen Union ist eine neue berufliche Herausforderung. Die länderübergreifende Zusammenarbeit mit den verschiedenen Mitgliedstaaten und Organen ist für mich ein großer Gewinn.

Der große Vorteil an meiner Tätigkeit ist die zeitliche Flexibilität. Die Arbeit lässt sich hervorragend mit unserem Familienleben in Einklang bringen. Die Bürotätigkeit ist immer in der Zeit, in der die Kinder im Kindergarten sind. In Belgien besteht der Vorteil, dass Ganztagsschulen und Kindergärten selbstverständlich sind. Die Kernzeiten sind von 9 bis 15 Uhr und die Möglichkeiten einer darüber hinausgehenden Betreuung fast unbegrenzt. Dadurch, dass der Großteil der Frauen in Belgien berufstätig ist, schließen sich die Mütter eng zusammen, um sich das Abholen der Kinder aufzuteilen, wenn es im Büro dann doch mal später wird. Ein weiterer Vorteil an diesem System ist, dass die Kinder in dieser Zeit alle wünschenswerten Freizeitaktivitäten erleben: Schwimmen gehen, Sportkurse, Musikunterricht ... und ganz nebenbei wachsen die Kinder dreisprachig auf. All dies wird in Belgien schon in Kindergärten und Schulen erledigt. Als Mutter von drei Kindern weiß ich das sehr zu schätzen, denn so müssen nicht jeweils zwei Kinder den Nachmittag im Auto verbringen, weil das andere Kind zu seinen Unternehmungen gefahren wird.

Es ist nicht von der Hand zu weisen, dass eine Berufstätigkeit neben der Familie mit drei kleinen Kindern organisatorische Höchstleistungen verlangt. Auch hinterlässt die körperliche Erschöpfung nicht selten seine Spuren, etwa wenn die Kinder krank sind und die Nächte wenig Schlaf bringen. Morgendliche Abschiedsgrüße meiner Kinder zum Beispiel in Form von Kakaoflecken an meiner Kleidung runden das Bild einer berufstätigen Mutter dann auch gänzlich ab. 